(C) 2008 The Japan Society of Applied Physics

\title{
Transmission Enhancement through Single Slit Embedded in Plasmonic Multilayer Structure
}

\author{
Chih-Ming Wang, Chien-Chang $\mathrm{CHAO}^{1}$, Hung-I HuAng ${ }^{1}$, Yia-Chung Chang, \\ Din Ping TsAI ${ }^{2}$, and Jenq-Yang CHANG ${ }^{1 *}$ \\ Research Center for Applied Sciences, Academia Sinica, Taipei, Taiwan 11529, R.O.C. \\ ${ }^{1}$ Department of Optics and Photonics, National Central University, Jhongli, Taiwan 32001, R.O.C. \\ ${ }^{2}$ Department of Physics, National Taiwan University, Taipei, Taiwan 106, R.O.C.
}

(Received January 13, 2008; accepted April 16, 2008; published online August 22, 2008)

The transmission properties of a plasmonic multilayer structure with a defect, which can be a nanoslit, were investigated. The transmission spectrum through the slit embedded in the plasmonic multilayer structure presents a Fano-like shape at a transmission peak of $580 \mathrm{~nm}$. The enhanced transmission is due to the localized surface plasmon resonant within the cavity of the plasmonic multilayer. The increased transmission factor has a maximum of 5.5 and it also performs loose angle tolerances of 10 and $20^{\circ}$ for 1 and $3 \mathrm{~dB}$ losses, respectively. [DOI: 10.1143/JJAP.47.6713]

KEYWORDS: surface plasmon waves, single slit, transmission enhancement, plasmonic multilayer

\section{Introduction}

Extraordinary transmission by subwavelength hole arrays ${ }^{1)}$ as well as a single hole surrounded by surface corrugations $^{2-5)}$ has attracted much interest and offers promising applications. The transmission processes of this type of grating-surrounded metallic aperture can be separated into three independent steps: coupling in, transmission through, and coupling out from the aperture. ${ }^{6,7)}$ The coupling in can be optimized by tailoring the geometric parameters for a higher transmission. ${ }^{2-4,8)}$ However, in current research, the input beam was considered as a plane wave with a single wave vector and an infinite beam width. Nevertheless, in practice, this type of device for visible light has only a size of about $10 \times 10 \mu \mathrm{m}^{2}$. Thus, the input beam should be focused for a higher optical efficiency. A focused beam has multiple wave vectors meaning that the device should have a loose angle tolerance. However, current grating-surrounded structures are highly angle-dependent owing to the nature of grating-coupled surface plasmon (SP). Thus, the transmission is limited. Recently, it has been found that two metallic layers with a nanogap, called the plasmonic multilayer structure, exert strong coupling effects.9' A localized surface plasmon (LSP) mode with an extremely large incidence angle tolerance was found. ${ }^{10}$ This kind of the angleindependent mode might be very useful to improve the small angle tolerance of transmission enhancement through a single aperture with surface corrugations. ${ }^{8)}$

In this study, we investigate the extraordinary transmission through a single slit embedded in the plasmonic multilayer structure that is composed of a metallic grating, a cavity layer, and a metallic film. The dispersion as well as the angle-resolved spectral response of the plasmonic multilayer structure is analyzed by the rigorous-coupled wave analysis (RCWA) method. ${ }^{11-13)}$ A resonant dip in reflectance with a high immunity for angle deviation is designed through the mechanism of the LSP. Following the calculation by the RCWA method, the transmission spectrum and the angle tolerance of a single nanoslit embedded in the plasmonic multilayer structure are analyzed by the finite differential time domain (FDTD) method. A Fano-like shape of transmission maximum is found in the spectrum at a wavelength of $580 \mathrm{~nm}$ with an increased transmission

*E-mail address: jychang@dop.ncu.edu.tw

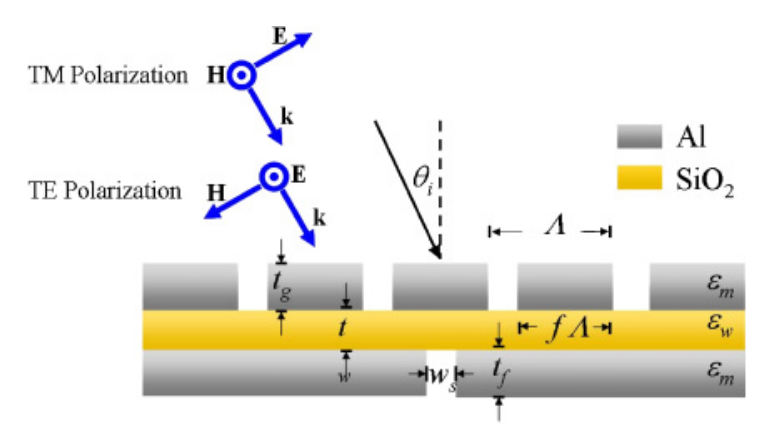

Fig. 1. (Color online) Basic geometry of investigated structure. It is a single slit embedded in the plasmonic multilayer structure consisting of a metallic grating, a cavity layer, and a metallic film.

factor maximum of 5.5. The enhanced transmission has loose angle tolerances of 10 and $20^{\circ}$ respectively for 1 and $3 \mathrm{~dB}$ losses. Finally, we also study the position tolerance of the nanoslit for the alignment to the grating in fabrication.

\section{Description for Simulation}

The proposed structure is presented in Fig. 1. It is a plasmonic multilayer structure composed of a metallic grating, a cavity layer, and a metallic film (from top to bottom), with a defect on the metallic film. In a twodimension structure, the defect can be a slit. The metallic grating periodicity is denoted by $\Lambda$, the filling factor is $f$ defined as the ratio of metal in a period, the thickness is denoted by $t_{\mathrm{g}}$, and its complex dielectric constant is denoted by $\varepsilon_{\mathrm{m}}$ where $\varepsilon_{\mathrm{m}}=\varepsilon_{\mathrm{mr}}+i \varepsilon_{\mathrm{mi}}$. Between and above the metallic grating, the dielectric constant is denoted by $\varepsilon_{\text {Air }}$. The thickness of the $\mathrm{SiO}_{2}$ cavity layer is assumed to be $t_{\mathrm{w}}$ and its dielectric constant is denoted by $\varepsilon_{\mathrm{w}}$. At the bottom, the material aluminum of the metallic film with a thickness of $t_{\mathrm{f}}$ is identical to that of the grating. The wavelengthdependent optical constants of the materials investigated above are obtained from ref. 14. A transverse electric (TE) or transverse magnetic (TM) polarized light is assumed to be launched on the metallic grating side with an incident plane wave at an angle $\theta_{\mathrm{i}}$. The input light is TM-polarized, in which the magnetic vector is oriented parallel to the grating grooves. We can find that the plasmonic multilayer structure (without a defect) is a periodic structure. To evaluate the optical property of the plasmonic multilayer structure 


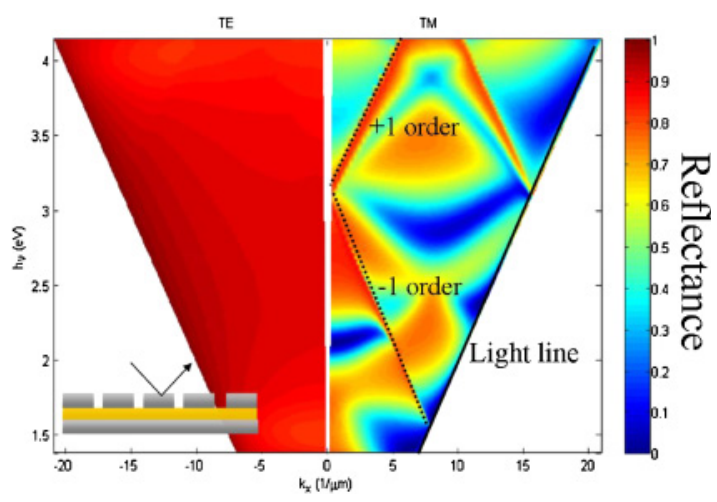

Fig. 2. (Color online) Angle-dependent reflectance of investigated structure simulated by RCWA method. A dark shading represents a lower reflectance while the incident light is confined inside the plasmonic multilayer structure.

quickly, we theoretically modeled it by the RCWA method. Compared with the FDTD method, the RCWA method is time-saving for theoretical evaluation of a periodic structure.

\section{Results and Analysis}

Figure 2 shows the simulated angle-dependent reflectance spectra obtained by the RCWA method and the inset is the simulated structure. A low reflectance is represented in darkblue shading, as shown by the color bar. The geometric parameters of the plasmonic multilayer structure are denoted by: $\Lambda=400 \mathrm{~nm}, f=0.825, t_{\mathrm{g}}=30 \mathrm{~nm}, t_{\mathrm{w}}=30 \mathrm{~nm}$, and $t_{\mathrm{f}}=110 \mathrm{~nm}$. In order to ensure that no energy will penetrate through the metallic film directly, the thickness of the metallic film at the bottom, $t_{\mathrm{f}}$, is set to be larger than $100 \mathrm{~nm}$. The left (TE-polarized) side and the right (TM-polarized) side of Fig. 2 present a very different feature. A high reflectance over the investigated spectral ranges from 1.4 to $4.1 \mathrm{eV}$ is shown when the TE-polarized light is applied. In contrast, the TM-polarized light presents a reflectance oscillation in both photon energy and in-plane wave vector dependence. This is because the grooves and the cavity layer are narrow. Therefore, the investigated frequencies are far below the cut-off frequency in the TE mode. ${ }^{15)}$ There is no TE guiding mode that can exist in such a narrow dielectric layer sandwiched in metals. At this time, the structure can be observed as a metallic surface with a small roughness. As a consequence, the reflectance of a TE-polarized light is always high, as shown on the left side of Fig. 2. Next, the spectrum shows a clear resonant dip occurring at $2.2 \mathrm{eV}$ for a TM-polarized light with a small incidence angle on the right side of Fig. 2. As the incidence angle increases, the resonant wavelength of the dip at $2.2 \mathrm{eV}$ is nearly the same for $0^{\circ}<\theta_{\mathrm{i}}<10^{\circ}$. At the same time, there are two peaks split from the resonant peak at $3.1 \mathrm{eV}$ that is plotted in black dots in the figure. The resonant peak at $3.1 \mathrm{eV}$ corresponds to the grating-coupled SP. On a metallic plate surface, the momentum of SP can be theoretically calculated by the equation $k_{\mathrm{SP}}=\sqrt{\varepsilon_{\mathrm{d}} \varepsilon_{\mathrm{m}} /\left(\varepsilon_{\mathrm{d}}+\varepsilon_{\mathrm{m}}\right)} k_{0}$, where $k_{0}$ is the wave vector in free space, $\varepsilon_{\mathrm{m}}$ and $\varepsilon_{\mathrm{d}}$ are the permittivity of the metal and the nearby dielectric, respectively. When the grating-coupled SP is excited, it must obey the conservation of the momentum for SP: $k_{\mathrm{SP}}=k_{x}+G$; where $k_{x}$ is the lateral wave vector of incidence light, $G=2 \pi \mathrm{m} / \Lambda$ denotes the reciprocal lattice vectors of the grating, $m$ is an integer and the two resonant peaks split from $3.1 \mathrm{eV}$ refer to $m=+1$ and -1 . Note that an interaction between the incident light and the grating/air interface produces the grating-coupled SP resonated at $3.1 \mathrm{eV}$. Thus, there is no energy transfer into the cavity layer. The incidence light transfer into the SP of the interface and later the SP re-radiates into the backward light, which forms a peak in reflectance spectra. However, the resonant dip at $2.2 \mathrm{eV}$ is relative to the LSP which is less affected by the incidence angle. A LSP of the metallic grating is excited by the incident light first and then the excited LSP couples with the SP on the interface between the $\mathrm{SiO}_{2}$ and $\mathrm{Al}$ films. That means that the energy can be guided in the cavity layer by the $\mathrm{SP}$ on the $\mathrm{SiO}_{2} / \mathrm{Al}$ interface. Thus, the plasmonic multilayer structure can confine the incident energy well with an angle tolerance of $10^{\circ}$.

Figure 3 shows the wavelength-dependent enhanced transmission factor of a nanoslit embedded in the plasmonic multilayer structure for a normally incident TM-polarized light that is simulated by the FDTD method. The geometric parameters were $\Lambda=400 \mathrm{~nm}, f=0.825, t_{\mathrm{g}}=30 \mathrm{~nm}, t_{\mathrm{w}}=$ $30 \mathrm{~nm}, t_{\mathrm{f}}=110 \mathrm{~nm}$, and $w_{\mathrm{s}}=50 \mathrm{~nm}$. The embedded slit is placed immediately under the center of the Al ridge. The enhanced transmission factor $\mathrm{F}$ is defined as the transmission power normalized to the width of the slit. Transmission power is numerically calculated using a circular monitor placed $1 \mu \mathrm{m}$ away from the exit of the slit. In Fig. 3, the transmission spectrum from 400 to $1100 \mathrm{~nm}$ shows a Fanolike shape and an increased transmission factor maximum of 5.5 at a wavelength of $580 \mathrm{~nm}$ corresponding to the resonant $\operatorname{dip}(2.2 \mathrm{eV})$ shown in Fig. 2 with a full-width at halfmaximum (FWHM) of $100 \mathrm{~nm}$.

The angle tolerance of this device is also analyzed at a wavelength of $580 \mathrm{~nm}$ and shown in Fig. 4(a). The 1 and $3 \mathrm{~dB}$ angle tolerances are 10 and $20^{\circ}$, respectively. The $H_{y}{ }^{2}$ distributions of the fields for $\theta_{\mathrm{i}}=0$ and $10^{\circ}$ were also simulated by the FDTD method at a wavelength of $580 \mathrm{~nm}$ and are shown in Figs. 4(b) and 4(c). Only a small shift of the field can be observed even when the incidence angle is modulated by $10^{\circ}$. Furthermore, the fields resonate in the lateral direction under the $\mathrm{Al}$ ridge. The resonant fields consist of three hot spots, which are the enhanced localized $H_{y}{ }^{2}$ fields, under each $\mathrm{Al}$

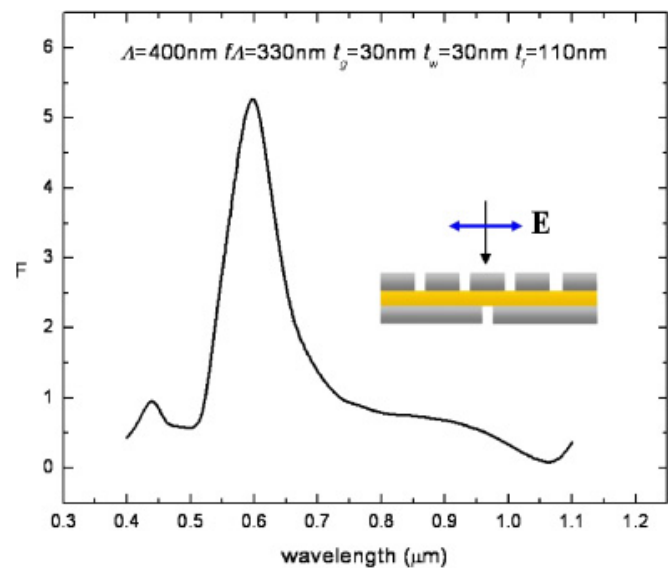

Fig. 3. (Color online) The enhanced transmission factor (F) of a single slit embedded in the plasmonic multilayer structure which is simulated by the FDTD method. The geometric parameters were $\Lambda=400 \mathrm{~nm}, f=$ $0.825, t_{\mathrm{g}}=30 \mathrm{~nm}, t_{\mathrm{w}}=30 \mathrm{~nm}, t_{\mathrm{f}}=110 \mathrm{~nm}$, and $w_{\mathrm{s}}=50 \mathrm{~nm}$. 


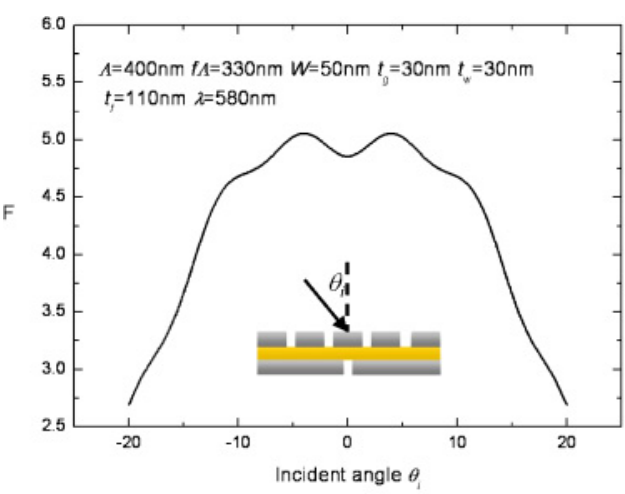

(a)

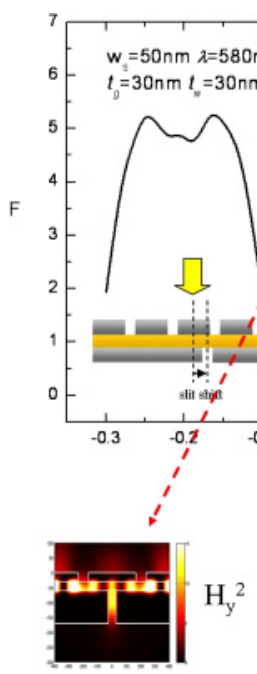

(b)

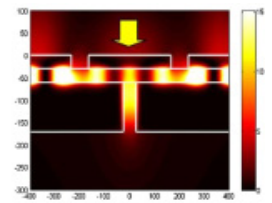

(b)

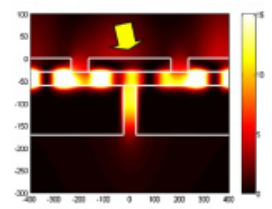

(c)
Fig. 4. (Color online) (a) Angular analysis of a single slit embedded in plasmonic multilayer structure. $H_{y}{ }^{2}$ field distribution at incident angles of (b) 10 and (c) $20^{\circ}$ immediately under the grating opening. Although, it also shows transmission maxima for the $\mathrm{SS}=160$ and $250 \mathrm{~nm}$. However, the optical properties for slit shifts of 160 and $250 \mathrm{~nm}$ are not discussed in this paper.

\section{Conclusions}

In summary, we investigate the extraordinary transmission through a single slit embedded in the plasmonic multilayer structure composed of a metallic grating, a cavity layer, and a metallic film. The transmission spectrum of the proposed structure presents a Fano-like shape and an enhanced transmission factor maximum of 5.5 at a wavelength of $580 \mathrm{~nm}$. The enhanced transmission has a loose angle tolerance of 10 and $20^{\circ}$ respectively for 1 and $3 \mathrm{~dB}$ losses. This type of device with a loose angle tolerance is suitable for enhancing the transmission of a focused beam through a single aperture.

\section{Acknowledgement}

The authors are grateful for the research support from the National Science Council of Taiwan, R.O.C., under project number NSC 95-2221-E-008-118-MY3.

1) T. W. Ebbesen, H. J. Lezec, H. F. Ghaemi, T. Thio, and P. A. Wolff: Nature 391 (1998) 667.

2) T. Thio, K. M. Pellerin, R. A. Linke, H. J. Lezec, and T. W. Ebbesen: Opt. Lett. 26 (2001) 1972.

3) T. Thio, J. J. Lezec, T. W. Ebbesen, K. M. Pellerin, G. D. Lewen, A Nahata, and R. A. Linke: Nanotechnology 13 (2002) 429.

4) F. J. Garcì-Vidal, H. J. Lezec, T. W. Ebbesen, and L. Martìn-Moreno Phys. Rev. Lett. 90 (2003) 213901.

5) H. J. Lezec and T. Thio: Opt. Express 12 (2004) 3629.

6) A. Degiron and T. W. Ebbesen: Opt. Express 12 (2004) 3694.

7) C. M. Wang, B. Ung, Y. Sheng, and J. Y. Chang: Opt. Commun. 265 (2006) 678.

8) C. M. Wang, H. I. Huang, C. C. Chao, J. Y. Chang, and Y. Sheng: Opt. Express 15 (2007) 3496

9) A. Christ, T. Zentgraf, S. G. Tikhodeev, N. A. Gippius, J. Kuhl, and H. Giessen: Phys. Rev. B 74 (2006) 155435.

10) C. M. Wang, Y. C. Chang, M. W. Tsai, Y. H. Ye, C. Y. Chen, Y. W Jiang, Y. T. Chang, S. C. Lee, and D. P. Tsai: Opt. Express 15 (2007) 14673.

11) M. G. Moharam and T. K. Gaylord: J. Opt. Soc. Am. 71 (1981) 811.

12) M. G. Moharam, E. B. Grann, D. A. Pommet, and T. K. Gaylord: J. Opt. Soc. Am. A 12 (1995) 1068.

13) L. Li: J. Opt. Soc. Am. A 13 (1996) 1024.

14) E. D. Palik: Handbook of Optical Constants (Academic, New York, 1985).

15) R. E. Collin: Field Theory of Guided Waves (IEEE, New York, 1991) 2nd ed., p. 329. 